Revista Electrónica Complutense de Investigación en Educación Musical ISSNe: 1698-7454

https://dx.doi.org/10.5209/reciem.59794

\title{
Diseño, implementación y evaluación de un programa para el aprendizaje de la guitarra en el grado de educación infantil a través de videotutoriales
}

\author{
Carlos Rodríguez Lozanoํㅜ, Gregorio Vicente Nicolás ${ }^{2}$
}

Recibido: 5 de abril de 2018 / Aceptado: 27 de mayo de 2019

Resumen. Los objetivos de este estudio han sido (1) diseñar e implementar un programa para el aprendizaje de la guitarra en el Grado en Educación Infantil a través de videotutoriales y (2) explorar las percepciones del alumnado y profesorado acerca de dicho programa. Su planteamiento metodológico se fundamenta en el visionado de videotutoriales antes y después de cada una de las seis sesiones de las que consta dicho programa El conjunto de participantes del estudio estuvo formado por el alumnado del último curso del Grado en Educación Infantil de la Universidad de Murcia $(\mathrm{N}=167)$ y dos profesores que llevaron a la práctica dicho programa. Para la recogida de información se elaboró un cuestionario ad hoc destinado al alumnado y una entrevista para los profesores. Los resultados revelaron que los participantes otorgan un papel fundamental a los videotutoriales en el proceso de aprendizaje desarrollado en este programa. Asimismo, el $75 \%$ del alumnado constató sentir cumplidas sus expectativas iniciales y se sintieron competentes para acompañar canciones con la guitarra en el aula de Educación Infantil.

Palabras clave: Educación Infantil; Educación Superior; formación instrumental; videotutoriales; programa de innovación educativa

\section{[en] Design, implement and evaluation of a program for learning to play the guitar in the Degree of Early Childhood Education through the use of video tutorials}

\begin{abstract}
The aims of this work has been (1) to design and implement a program for learning to play the guitar in the Degree of Early Childhood Education through the use of video tutorials and (2) explore the perceptions of the students and teachers about that program. Its methodological approach is based on the viewing of video tutorials before and after each of the six sessions of which this program consists. The sample of participants of the study was formed by the students of the last year of the Degree in Early Childhood Education of the University of Murcia $(\mathrm{N}=167)$ and two teachers who put this program into practice. For the data collection an ad hoc questionnaire was designed for the students and an interview for the teachers. The results revealed that the participants give a fundamental role to the video tutorials in the learning process developed in this program. Likewise, $75 \%$ of students verified that their initial expectations were met and they felt competent to accompany songs with the guitar in the Preschool classroom.
\end{abstract}

Keywords: Early Childhood Education; Higher Education; instrumental training; video tutorials; educational innovation programme

Sumario. 1. Introducción. 2. Método. 2.1. Contexto y participantes. 2.2. Instrumentos. 2.3. Procedimiento. 2.4. Análisis de datos. 3. Resultados. 3.1. Aspectos formales del programa. 3.2. Aspectos

\footnotetext{
$1 \quad$ Facultad de Educación. Universidad de Murcia (España)

E-mail: carlos.rodriguez2@um.es

2 Facultad de Educación. Universidad de Murcia (España)

E-mail: gvicente@um.es
} 
docentes. 3.3. Análisis de los datos obtenidos en la entrevista. 3.3.1. Aspectos generales del programa. 3.3.2. Aspectos docentes. 4. Conclusiones. 5. Referencias bibliográficas.

Cómo citar: Rodríguez-Lozano, C. y Vicente-Nicolás, G. (2019). Diseño e implementación de un programa para el aprendizaje de la guitarra en el grado de educación infantil a través de videotutoriales. Revista Electrónica Complutense de Investigación en Educación Musical, 16, 95-115.

\section{Introducción}

La formación musical del profesorado de Educación Infantil ha estado presente prácticamente en la totalidad de planes de estudios vigentes en las últimas décadas en las universidades españolas (Lorenzo, 1995). Dicha formación incluye la interpretación musical con instrumentos escolares y, en muchos casos, el dominio de la flauta dulce, pero en ningún caso, salvo alguna excepción, el de instrumentos polifónicos que permitan acompañar el canto. La Orden ECI/3854/2007 del 27 de diciembre, por la que se establecen los requisitos para la verificación de los títulos universitarios oficiales que habiliten para el ejercicio de la profesión de Maestro en Educación Infantil y, más concretamente en el módulo de Música, Expresión Plástica y Corporal, no se presenta ninguna competencia específica relacionada con la formación instrumental del alumnado de esta titulación. Sin embargo es frecuente el uso de instrumentos de pequeña percusión o la flauta dulce, herencia de los diferentes métodos musicales introducidos en España por Escudero, Murcia Sanuy y Elizalde (Jambrina, 2007).

Este estudio parte de las dificultades que conlleva el aprendizaje de un instrumento polifónico en contextos de Educación Superior, concretamente en los estudios de Grado en Educación Infantil. La inclusión del aprendizaje de un instrumento polifónico supone un aumento considerable del nivel de competencia musical que se exige al alumnado, nivel que se encuentra limitado en muchas ocasiones por algunos condicionantes de las asignaturas de música de estas titulaciones (el número reducido de créditos, el volumen de contenidos disciplinares y didácticos que deben impartirse, el nivel tan básico de conocimientos musicales previos, una ratio elevada...) y que podrían impedir alcanzar los objetivos óptimos deseados. Si se compara con la formación instrumental individualizada que recibe el alumnado en centros de música o conservatorios, el aprendizaje de un instrumento polifónico en el contexto universitario español del Grado de Educación Infantil es todo un reto, tanto para el profesorado, como para el alumnado. Si atendemos a sus programas y guías docentes, es cierto que los objetivos y exigencias no son los mismos que los de un conservatorio, pero no es menos cierto que el profesorado especialista de Educación Infantil debe ofrecer a su alumnado ejemplos musicales de calidad, lo que implica un dominio aceptable del instrumento que se practica.

Paralelamente a la situación desfavorable descrita inherente al aprendizaje instrumental en los espacios universitarios, no debe ignorarse una temática estrechamente relacionada como es la formación musical del profesorado de Educación Infantil. Autores como Bernal (1996), López de la Calle (2009) o Ruiz y Santamaría (2013) señalan que los docentes de esta etapa muestran un sentimiento generalizado de falta de formación musical y, en consecuencia, abogan por la participación de un especialista de música en la docencia de este arte es sus aulas. La falta de cualificación y de confianza en sí mismos para enseñar música es ratificada por otros investigadores como Giles y Frego (2004), Gifford (1993) o Mills (2005). Además, los estudios rea- 
lizados sobre la educación musical en el currículo de Educación Infantil dan muestras de que la implementación es mínima, incluso relegando la música a un papel secundario o casi testimonial (Giráldez, 2009).

Las limitaciones temporales, organizativas y formativas, podrían inducir, o inducen en ocasiones, a relegar al olvido algunas aptitudes fundamentales que todo el profesorado de Educación Infantil debería poseer para favorecer el desarrollo de las potencialidades musicales de su alumnado (Giráldez, 2010). Entre ellas y siguiendo Vannatta-Hall (2010), se encuentran el oído musical, el sentido del ritmo, la entonación de la voz e, íntimamente relacionada con este estudio, la práctica instrumental. De igual modo, Capriles (2016) enfatiza la necesidad de incluir en la formación musical del profesorado de infantil y de primaria instrumentos de fácil acceso y coste asequible que permitan lograr los objetivos más básicos e imprescindibles desde el punto de vista instrumental. Esta autora propone en su estudio la utilización del guitarró u otros instrumentos similares, como podría ser la guitarra.

Junto a los argumentos planteados, el proceso de adaptación y convergencia al Espacio Europeo de Educación Superior en el que la universidad española se encuentra inmersa desde hace años, invita a tomar como referentes otros modelos formativos en donde las maestras y maestros de Educación Infantil deben ser competentes para acompañar las canciones que interpretan en sus aulas. Sirvan como ejemplos países como Letonia, Finlandia, Polonia o, en contextos no europeos, Japón.

No es necesario justificar que las actividades de canto que las maestras y maestros de infantil plantean en sus aulas adquieren una mayor dimensión musical si cuentan con un acompañamiento instrumental. Por este motivo, en el momento de inicio del presente estudio relacionado con la formación musical de este profesorado, se consideró que la guitarra podría ser uno de los instrumentos más apropiados para desempeñar tal función, principalmente porque, a diferencia de otros instrumentos como la flauta, permite cantar y tocar al mismo tiempo, además de su accesibilidad económica.

Siendo consecuentes con las dificultades que surgen en el aprendizaje de este instrumento en el contexto de la Educación Superior, se consideró pertinente plantear un programa para el aprendizaje de la guitarra a partir de las oportunidades y el avance tecnológico de las TIC en los últimos años que aminorase las limitaciones anteriormente planteadas. Un ejemplo de esta evolución educativa y tecnológica es la aparición de un recurso prácticamente indispensable para el alumno: el videotutorial (vídeo educativo). Bengochea, Domínguez y Díez (2014) definen el videotutorial como "un vídeo que trata de enseñar algo a través de la percepción dual: escuchar y ver. Traduce el contenido en sonidos e imágenes y utiliza elementos de la expresión audiovisual [...], dependiendo del tipo de enseñanza-aprendizaje al que está destinado" (p. 28). Entre los diferentes beneficios que este recurso proporciona, Ródenas (2012) argumenta los siguientes: (1) muestra paso a paso los procedimientos a seguir para elaborar una actividad; (2) permite al estudiante acceder a él en cualquier momento y cuantas veces lo necesite; (3) facilita la comprensión de los contenidos más difíciles para los estudiantes; (4) y facilita la atención personalizada del alumno.

El uso del videotutorial en contextos educativos ha evolucionado considerablemente en las últimas décadas, al mismo tiempo que han aumentado las aplicaciones que de este recurso se han realizado en al ámbito del aprendizaje músico-instrumental. Entre algunos de los trabajos desarrollados en esta línea se encuentra aquel realizado por Bautista (2000), quien diseñó un método audiovisual (MAEI) para el estudio técnico-interpretativo del timple canario. Este autor utilizó vídeos en los cuales apa- 
recían imágenes estáticas con breves explicaciones de los ejercicios que el alumnado debía realizar. Otros autores como Navarro, Lavigne y Martínez (2009), desarrollaron un curso para la iniciación en la interpretación de la guitarra clásica a través de una plataforma web (blog) y una serie de herramientas complementarias, entre las que se encontraban videotutoriales. Igualmente, Shoemaker y Van Stam (2010) llevaron a cabo lecciones de piano desde Norte América hasta África mediante la distribución sincrónica y asincrónica de videotutoriales. Esta combinación de modalidad de visionado permitía minimizar los problemas provocados por la baja velocidad de internet en algunos países africanos. Para el mismo instrumento, Upitis, Brook y Abrami (2014) realizaron una prueba con la plataforma iScore con un alumno pianista de 15 años. Esta herramienta les permitió hacer anotaciones de vídeo o audio con comentarios sobre la interpretación musical que previamente se había grabado. Esta función de anotación admitía hacer observaciones tanto al profesor como al alumno. En contextos escolares, Palazón (2013) realizó una investigación basada en la aplicación de podcast de vídeo y audio aplicados a la interpretación musical de la flauta dulce con alumnado de $4^{\circ}$ curso de Educación Secundaria Obligatoria, obteniendo resultados muy positivos.

Todos los estudios mencionados confirman la idoneidad del videotutorial para el aprendizaje instrumental. Sin embargo y como afirma López y Lago (2013), todavía existen dudas referentes a la calidad y resultados de la enseñanza musical a distancia y, especialmente, dedicada a la interpretación musical. Asimismo, sorprende la falta de una mayor cantidad de experiencias investigadoras en relación con esta temática Lockett (2010).

De igual modo, no se debe olvidar que en el proceso de enseñanza-aprendizaje de un instrumento a través de las TIC el rol del docente es un elemento de gran importancia. La estrategia metodológica y el tipo de comunicación entre el profesoralumno durante el proceso incidirá en el grado de éxito de éste (Area, 2007). En esta misma línea, Sergio Blardony, entrevistado por Giráldez (2012), enfatiza la necesidad en una docencia vocacional y de compromiso por parte del profesor junto a un seguimiento profundo en los niveles iniciales del aprendizaje de un instrumento. Así mismo, como afirma Espigares, García, Tejada y Rebollo (2014) junto a una apropiada formación, condiciona que el docente pueda superar las diferentes dificultades y haga de este tipo de modelo de enseñanza fundamentado en la utilización del videotutorial como herramienta TIC, una nueva vía de comunicación y estrategia metodológica para el aprendizaje de un instrumento musical

Partiendo de las ideas expuestas anteriormente y con la finalidad de dar respuestas a determinados problemas suscitados en relación con la formación instrumental del profesorado de Educación Infantil, los objetivos de este estudio han sido: (1) diseñar e implementar un programa para el aprendizaje de la guitarra en el Grado en Educación Infantil a través de videotutoriales y (2) explorar las percepciones del alumnado y profesorado acerca de dicho programa.

\section{Método}

Este estudio está fundamentado en el modelo de diseño y desarrollo de investigación tecnológica educativa de Reeves (2000; 2006), quien articula este proceso en ciclos de diseño, validación, análisis y rediseño para una mejora y perfeccionamiento de la intervención e investigación (Figura 1). En el primer ciclo se analizó el problema y se hizo una revisión de la literatura científica, en este caso, aquella relacionada con la formación instrumental de los 
futuros maestros y maestras de Educación Infantil. A continuación, se planteó una posible solución fundamentada teóricamente, que se materializó en el diseño de un programa para el aprendizaje de la guitarra a través del uso de videotutoriales. Posteriormente, se llevaron a cabo los diferentes programas mediante una experiencia piloto inicial (2012-2013) y el propio Curso de iniciación a la guitarra en Educación Infantil (2013-2014). Finalmente se procedió a la recogida de información y evaluación del proceso de aplicación del programa con el fin de obtener unos principios estructurales y de diseño para este modelo de aprendizaje.

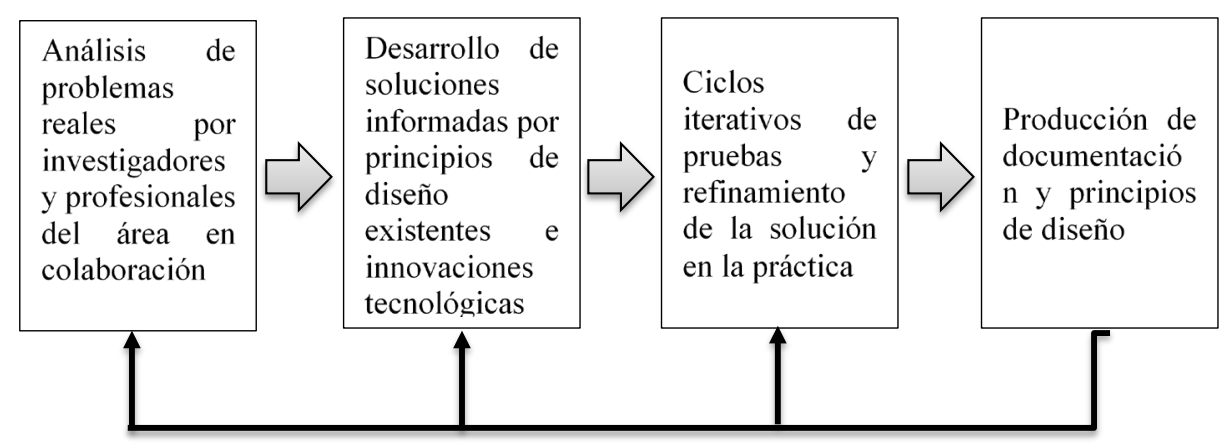

Figura 1. Diseño del método de investigación basado en la investigación de Tecnología Educativa (Reeves, 2006)

\subsection{Contexto y participantes}

Los participantes de este estudio han sido los alumnos y alumnas de los tres grupos del Grado en Educación Infantil que cursaban la asignatura de Percepción y Expresión Musicales ( $4^{\circ}$ curso) en la Facultad de Educación de la Universidad de Murcia durante el año académico 2013-2014. Para su selección se ha utilizado un muestreo no probabilístico y dentro de esta categoría el muestreo por conveniencia, atendiendo a la accesibilidad que se tenía a los participantes y el deseo de los mismos de formar parte del estudio (McMillan y Schumacher, 2005). El total de la muestra asciende a 167 participantes, que se corresponde con un 74.2\% de total del alumnado matriculado en esa asignatura. E1 99\% de la muestra eran mujeres de edades comprendidas entre los 21 y 25 años. El 86\% de los participantes admitió no tener conocimientos de guitarra. El 14\% restante lo adquirió a través de: clases particulares (5.2\%), conservatorio (0.5\%), Educación Primaria (2.1\%), Educación Secundaria Obligatoria $(3,6 \%)$, Bachillerato $(0.5 \%)$ y de forma autodidacta $(1.6 \%)$. Asimismo, en una escala del 1 al 10, solo un 3\% indicó que su nivel de conocimiento de guitarra era $\geq$ a 5 .

\subsection{Instrumentos}

Para este estudio se han utilizado dos instrumentos de recogida de información, uno para el alumnado participante y otro para el profesorado. El primero de ellos ha consistido en la elaboración de un cuestionario ad hoc con preguntas cerradas de carácter dicotómico y politómico, con escalas de valoración tipo Likert de 4 puntos (1: muy en desacuerdo; 2: en desacuerdo; 3: de acuerdo; 4: totalmente de acuerdo), a excepción de los datos personales y formación musical. El cuestionario fue validado por el Panel Internacional de Investigación en Tecnología Educativa y se estructuró en los siguien- 
tes apartados: (1) Datos personales y formación musical; (2) Educación Musical en Educación Infantil; (3) Aptitudes musicales; (4) Evaluación del Curso de iniciación a la guitarra en Educación Infantil; (5) Tecnologías para la enseñanza de la música en Educación Superior; y (6) Evaluación de los recursos audiovisuales del curso. A continuación, se indican los ítems de cada uno de los apartados analizados en este trabajo:

1. Datos personales y formación musical:

- Sexo.

- Edad.

- Conocimientos musicales y guitarrísticos.

2. Educación Musical en Educación Infantil:

- Valoración de la guitarra como instrumento apropiado en Educación Infantil.

3. Aptitudes musicales:

- Mejora de la formación musical e instrumental a través del vídeo en red.

- Capacidad para acompañar canciones infantiles con la guitarra.

4. Evaluación del Curso de iniciación a la guitarra en Educación Infantil.

- Proyección del aprendizaje de la guitarra en el desarrollo futuro de la docencia en Educación Infantil.

- Consideración de las TIC y la Web 2.0 como elementos que potencian el proceso de enseñanza-aprendizaje musical.

- Valoración de la metodología.

- Importancia del docente en el proceso de enseñanza-aprendizaje.

- Motivación del profesorado a través de los recursos audiovisuales.

- Exigencia mostrada por el profesorado a través de los recursos audiovisuales.

- Número de sesiones presenciales y distribución temporal satisfactoria.

- Expectativas iniciales cumplidas.

5. Tecnologías para la enseñanza de la música en Educación Superior (no se incluyen ítems en este trabajo).

6. Evaluación de los recursos audiovisuales del curso.

- Indispensabilidad de los recursos audiovisuales disponibles en el curso.

- Motivación generada por el uso de los videotutoriales en el aprendizaje personal del alumnado.

El segundo de los instrumentos utilizados ha sido una entrevista semiestructurada destinada a los otros dos profesores responsables de la asignatura que implementaron el programa y que no habían participado en su elaboración. Con ella se pretendía conocer la opinión del profesorado sobre los materiales utilizados, la implementación del programa y aspectos relacionados con la metodología. A partir del guion establecido y de las temáticas planteadas espontáneamente por los entrevistados, se definieron las siguientes categorías de contenido.

a) Valoración de la experiencia como docentes

b) Planificación del programa de intervención: contenidos, número y distribución temporal de las sesiones

c) Videotutoriales: calidad de imagen, calidad de audio, discurso, estructuración y claridad 
d) Proceso de enseñanza-aprendizaje mediado por el uso de TIC

\subsection{Procedimiento}

$\mathrm{Al}$ inicio de la investigación se consideró indispensable realizar un programa piloto que permitiese su ulterior refinamiento y rediseño. De esta forma, se elaboró un primer progra-

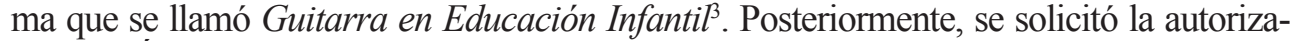
ción del Área de Música y Didáctica de la Expresión Musical de la Universidad de Murcia para implementar dicho programa piloto en el curso académico 2012/2013, así como el consentimiento del alumnado participante (un grupo de clase, $\mathrm{N}=65$ ). Ambos colectivos fueron informados sobre todos los aspectos relacionados con la investigación. El programa fue implementado por el profesor que lo había diseñado. Finalizada la experiencia piloto, se planteó la versión definitiva que se denominó Curso de iniciación a la guitarra en Educación Infantil. Con tal de implementar la versión definitiva del programa, se solicitó nuevamente el consentimiento al alumnado participante (curso 2013/2014), que en esta ocasión procedía de tres grupos $(\mathrm{N}=167)$ debido a que todos los profesores $(\mathrm{N}=3)$ que impartían la asignatura de Percepción y Expresión Musicales ( $4^{\circ}$ curso-Grado en Educación Infantil) lo pusieron en práctica en sus respectivos grupos ${ }^{4}$. Al inicio y al final del programa piloto y del definitivo se aplicaron cuestionarios al alumnado participante cuya finalidad era recabar información sobre diferentes aspectos mencionados en el apartado de anterior, tales como: datos personales, aptitudes musicales, valoración del "curso" y de los materiales, entre otros. Asimismo, en el programa definitivo también se realizó una entrevista a los otros dos profesores responsables de la asignatura que lo implementaron y que no habían participado en su elaboración. Se efectuaron grabaciones de audio de las entrevistas, que fueron transcritas posteriormente por los investigadores.

\section{a) Programa piloto: Guitarra en Educación Infantil}

Como se ha mencionado anteriormente, durante el curso académico 2012-2013 se realizó una experiencia piloto en un solo grupo que permitió un primer acercamiento a la realidad en donde se iba a desarrollar el posterior programa, así como la puesta en práctica de algunos materiales elaborados $a d$ hoc. Este programa se estructuró en seis sesiones teniendo en cuenta la asignación de horas que se había establecido para estos contenidos en el conjunto de la asignatura. Todas las sesiones se desarrollaron en un cuatrimestre. El conjunto de materiales diseñados estaba compuesto por siete videotutoriales, diferentes documentos teóricos sobre los contenidos trabajados y los audios de las canciones del repertorio interpretado. Para facilitar el acceso a los mismos, se creó la web Guitarra en Educación Infantil destinada a todo el alumnado participante.

Evaluada la puesta en práctica del programa piloto se extrajeron los siguientes criterios técnicos y didácticos para la reelaboración de los videotutoriales:

- Reducir la duración de los videotutoriales al igual que la carga excesiva de formas, colores y fuentes diferentes

- Mejorar el diseño de los videotutoriales y crear una unidad de formato que resultara atractiva

- Priorizar el aspecto didáctico sobre el aspecto técnico

http://carlosrola1990.wix.com/guitarrainfantil

Uno de los profesores era el creador del Curso de iniciación a la guitarra. 
- Mejorar la calidad de imagen y de sonido para facilitar una correcta percepción de los ejemplos incluidos en los materiales

En relación con la temporalización del programa, los participantes constataron la necesidad de aumentar el número de sesiones y su distribución a lo largo de todo el curso.

Los errores y aciertos resultantes de la evaluación fueron el punto de partida del rediseño de los nuevos videotutoriales y materiales que se implementarían en el programa definitivo (2013-2014).

b) Programa definitivo: Curso de iniciación a la guitarra en Educación Infantil

Este programa se estructuró igualmente en seis sesiones y no se pudo aumentar su número, debido a que las horas destinadas a la práctica de la guitarra estaban determinadas por la distribución de contenidos de la asignatura. Sin embargo, las sesiones se dispusieron a lo largo de los dos cuatrimestres para ofrecer al alumnado más tiempo para asimilar los procedimientos musicales. Cada sesión constaba de: (1) objetivos, (2) contenidos, (3) propuesta de actividades previas y de calentamiento, (4) actividades de desarrollo y profundización de los aspectos trabajados previamente con los videotutoriales y (5) tareas posteriores y de repaso. Asimismo, se detallaba la temporalización de las actividades, los documentos necesarios que se iban a utilizar y los videotutoriales que debían visionarse antes y después de la sesión (ver ejemplo de sesión en Anexo 1). Los contenidos trabajados en todas las sesiones y el número de vídeos realizados para cada una se presentan en la Tabla 1.

Tabla 1. Contenidos de las sesiones del Programa de Iniciación a la Guitarra.

\begin{tabular}{|c|c|}
\hline $\begin{array}{l}\text { Sesión } 1 \\
\text { Keep calm and play the gui- } \\
\text { tar } \\
\text { ( } 5 \text { vídeos) }\end{array}$ & $\begin{array}{l}\text { - } \text { Cuerdas al aire de la guitarra para afinar y para las notas de referencia } \\
\text { - } \text { Partes de la guitarra } \\
\text { - } \text { Posición de la guitarra. Las manos en la guitarra } \\
\text { - Canciones: Vamos a palmear, ¿Dónde estás? }\end{array}$ \\
\hline $\begin{array}{l}\text { Sesión } 2 \\
\text { Todo es comenzar } \\
(10 \text { vídeos })\end{array}$ & $\begin{array}{l}\text { - Conocimientos de la nomenclatura general de los acordes } \\
\text { - Acordes: Am, G, G7 } \\
\text { - Canciones: Mariposa revoltosa, El cuco, Con mis manitas, Din-dón }\end{array}$ \\
\hline $\begin{array}{l}\text { Sesión } 3 \\
\text { Cuestión de práctica } \\
(6 \text { vídeos) }\end{array}$ & $\begin{array}{ll}\text { - } & \text { Acordes: E y Em } \\
\text { - } & \text { Cambio entre acordes: Am-Em. C-Em } \\
\text { - } & \text { Canciones: Sobre un pino verde, Sal Solecito, Las agujas del Reloj }\end{array}$ \\
\hline $\begin{array}{l}\text { Sesión } 4 \\
\text { Cada día cuenta } \\
\text { ( } 7 \text { vídeos) }\end{array}$ & $\begin{array}{l}\text { - } \text { Acordes: A y Dm } \\
\text { - } \text { Cambio entre acordes: G-D, A-D y D-E } \\
\text { - } \text { Cegundo rasgueo binario } \\
\text { Los monos: Los patitos, Cómo planta usted las flores, Hop hop hop, }\end{array}$ \\
\hline $\begin{array}{l}\text { Sesión } 5 \\
\text { Aprieta fuerte } \\
(4 \text { vídeos })\end{array}$ & $\begin{array}{l}\text { - } \text { Acordes: F } \\
\text { - Cambio entre acordes: C-F } \\
\text { - Canciones: Luna lunera, Las hormigas van de paseo, Aserrín }\end{array}$ \\
\hline $\begin{array}{l}\text { Sesión } 6 \\
\text { Disfruta cada nota } \\
(5 \text { vídeos) }\end{array}$ & $\begin{array}{l}\text { - } \text { Todos los acordes } \\
\text { - } \text { Rasgueo ternario } \\
\text { - } \text { cejilla fija } \\
\text { - Canciones: Cucú cantaba la rana, Pimpón, El cuco }\end{array}$ \\
\hline
\end{tabular}


Con respecto a los nuevos videotutoriales del programa, tras una profunda revisión de aquellos elaborados en el programa piloto y atendiendo a los criterios resultantes de la evaluación llevada a cabo, se diseñaron 37 videotutoriales de menor duración. Estos nuevos materiales contaban con una calidad de imagen-sonido ${ }^{5}$ superior, una mayor fluidez en el discurso y una mejor estructura para su comprensión y asimilación. Además, se integraron algunas novedades entre las que se destacan:

- Inclusión de una recapitulación final con los contenidos trabajados y recomendaciones para el trabajo autónomo con la finalidad de destacar las ideas más importantes del videotutorial y guiar el trabajo posterior del alumnado. La estructura de los vídeos quedó de la siguiente forma: introducción, desarrollo y recapitulación.

- Incorporación de planos cercanos que permitieran una mejor visualización de cuestiones técnicas (rasgueos y acordes).

- Inclusión de cierto grado de transparencia en las imágenes incorporadas en los videotutoriales para favorecer una mejor visualización de todos los elementos en la pantalla (Figura 2).

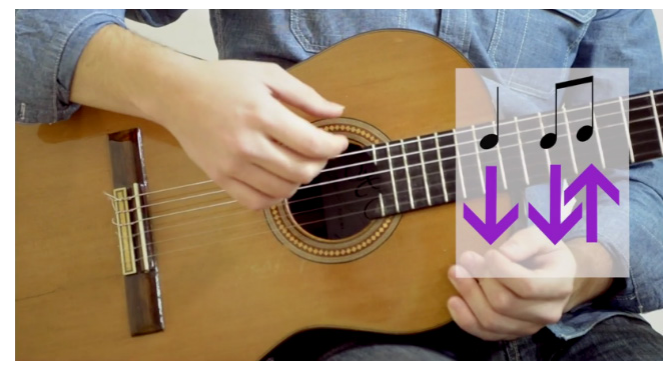

Figura 2. Captura de pantalla videotutorial: rasgueo con transparencia.

- Posicionamiento inferior con ajuste central de la partitura en la pantalla del videotutorial, así como la inclusión de una marca de color móvil que permitiera el seguimiento de la canción interpretada por el profesor. De igual forma, este seguimiento de la partitura estaba sincronizado con la aparición de las flechas que marcaban el sentido del rasgueo (Figura 3).

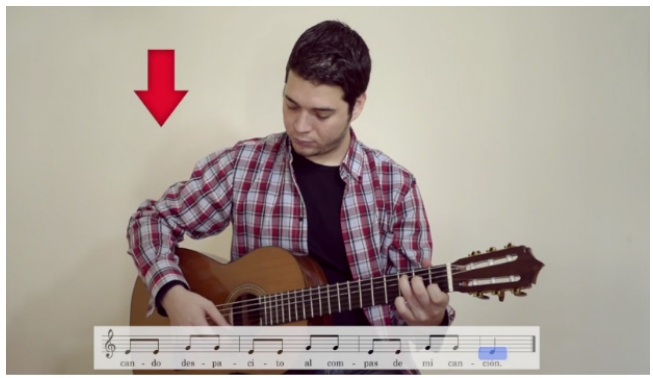

Figura 3. Captura de pantalla videotutorial: partitura con marcadores móviles.

Esto fue posible gracias a una mayor calidad de recursos para la grabación de la imagen y sonido: cámaras réflex (Nikon D600; Nikon D3100), objetivos fotográficos (50mm F/1.4; 24-70mm F/2.8G), luz artificial (2 pantallas Aputure Amaran 190C) y grabadora digital Zoom H1. 


\subsection{Análisis de datos}

Para el análisis de datos de tipo cuantitativo se ha utilizado el programa estadístico SPSS (vs.19). Para todos los ítems del cuestionario se calcularon diferentes estadísticos descriptivos como frecuencia, porcentaje, media y desviación típica. La información cualitativa que han aportado las entrevistas se ha analizado siguiendo el método de la teoría fundamentada (Grounded Theory- GT), coherente con la propuesta de Miles y Huberman (1994) adaptada por Serrano (1999). La GT se caracteriza por su poder explicativo de conductas humanas dentro de un campo de estudio concreto. Las fases que se plantean en la GT son: categorización (agrupamiento de casos), codificación (identificación de todas las categorías), comparación de categorías, "saturación teórica" (no se pueden identificar más categorías) y, por último, definición de las mismas. Las categorías de este estudio se especifican en el apartado de resultados.

\section{Resultados}

En este apartado se presentan los resultados más destacados de la investigación relacionados con el programa definitivo: Curso de iniciación a la guitarra en Educación Infantil. Se articulan en dos grandes bloques: (1) aspectos generales del programa y (2) actuación docente. En un primer momento se comenta la información proporcionada por los cuestionarios y, posteriormente, aquella procedente de las entrevistas.

\subsection{Aspectos generales del programa}

Una de las novedades de este curso de formación instrumental había sido la inclusión de la guitarra como instrumento acompañante de canciones en contextos de Educación Infantil. Al respecto, un 80.9\% estuvo de acuerdo o totalmente de acuerdo con la conveniencia de dicho instrumento para acompañar el canto en el aula de Infantil (Tabla 2). No se observaron diferencias importantes entre los valores obtenidos por los distintos grupos.

Tabla 2. La guitarra puede ser un instrumento muy apropiado en Educación Infantil.

\begin{tabular}{|l|c|c|c|c|c|c|c|c|c|c|}
\hline $\mathrm{N}=167$ & \multicolumn{2}{|c|}{1} & \multicolumn{2}{|c|}{2} & \multicolumn{2}{|c|}{3} & \multicolumn{2}{|c|}{4} & \\
\hline Grupo & $\mathrm{F}$ & $\%$ & $\mathrm{~F}$ & $\%$ & $\mathrm{~F}$ & $\%$ & $\mathrm{~F}$ & $\%$ & Media & D.Típ \\
\hline $\mathrm{A}$ & 1 & 2.3 & 11 & 25.0 & 30 & 68.2 & 2 & 4.5 & 2.75 & 0.576 \\
\hline $\mathrm{B}$ & 2 & 3.8 & 10 & 19.2 & 35 & 67.3 & 5 & 9.6 & 2.83 & 0.648 \\
\hline $\mathrm{C}$ & 0 & 0 & 8 & 11.3 & 53 & 74.6 & 10 & 14.1 & 3.03 & 0.506 \\
\hline Total & 3 & 1.8 & 29 & 17.4 & 118 & 70.7 & 17 & 10.2 & 2.89 & 0.581 \\
\hline 1= Muy en desacuerdo; 2 En desacuerdo; 3 De acuerdo; 4= Totalmente de acuerdo \\
\hline
\end{tabular}

En relación con la posible mejora de la formación musical e instrumental del futuro profesorado de Educación Infantil a través del "vídeo en red", un 92.2\% consideró que este programa había favorecido su formación musical e instrumental. El análisis por grupos no reveló resultados muy diferenciados (Tabla 3). 
Tabla 3. Mejora de la formación musical e instrumental mediante la utilización del "vídeo en red".

\begin{tabular}{|l|c|c|c|c|c|c|c|c|c|c|}
\hline $\mathrm{N}=167$ & \multicolumn{2}{|c|}{1} & \multicolumn{2}{|c|}{2} & \multicolumn{2}{|c|}{3} & \multicolumn{2}{|c|}{4} & \\
\hline Grupo & $\mathrm{F}$ & $\%$ & $\mathrm{~F}$ & $\%$ & $\mathrm{~F}$ & $\%$ & $\mathrm{~F}$ & $\%$ & Media & D.Típ \\
\hline A & 2 & 4.5 & 5 & 11.4 & 25 & 56.8 & 12 & 27.3 & 3.07 & 0.759 \\
\hline $\mathrm{B}$ & 0 & 0 & 4 & 7.7 & 35 & 67.3 & 13 & 25.0 & 3.17 & 0.550 \\
\hline C & 0 & 0 & 2 & 2.8 & 49 & 69.0 & 20 & 28.2 & 3.25 & 0.499 \\
\hline Total & 2 & 1.2 & 11 & 6.6 & 109 & 65.3 & 45 & 26.9 & 3.18 & 0.594 \\
\hline
\end{tabular}

1= Muy en desacuerdo; $2=$ En desacuerdo; $3=$ De acuerdo; $4=$ Totalmente de acuerdo

E1 95.8\% del alumnado consideró indispensables para su aprendizaje de la guitarra los recursos audiovisuales del curso de iniciación a la guitarra. Los resultados grupales no presentaron diferencias considerables (Tabla 4).

Tabla 4. Indispensabilidad de los recursos audiovisuales del curso de iniciación a la guitarra.

\begin{tabular}{|l|c|c|c|c|c|c|c|c|c|c|}
\hline N=167 & \multicolumn{2}{|c|}{1} & \multicolumn{2}{|c|}{2} & \multicolumn{2}{c|}{3} & \multicolumn{2}{c|}{4} & \\
\hline Grupo & $\mathrm{F}$ & $\%$ & $\mathrm{~F}$ & $\%$ & $\mathrm{~F}$ & $\%$ & $\mathrm{~F}$ & $\%$ & Media & D. Típ \\
\hline A & 0 & 0 & 2 & 4.5 & 21 & 47.7 & 21 & 47.7 & 3.43 & 0.587 \\
\hline B & 0 & 0 & 3 & 5.8 & 31 & 59.6 & 18 & 34.6 & 3.29 & 0.572 \\
\hline C & 0 & 0 & 2 & 2.8 & 20 & 28.2 & 49 & 69.0 & 3.66 & 0.533 \\
\hline Total & 0 & 0 & 7 & 4.2 & 72 & 43.1 & 88 & 52.7 & 3.49 & 0.579 \\
\hline
\end{tabular}

En cuanto a la motivación que ha generado el uso de los videotutoriales en el alumnado de este curso, un $85.6 \%$ manifestó haberse sentido motivado gracias a la utilización de estos videotutoriales (Tabla 5). Los valores obtenidos por cada grupo no mostraron diferencias importantes.

Tabla 5. Motivación generada por el uso de los videotutoriales en el aprendizaje personal del alumnado

\begin{tabular}{|l|c|c|c|c|c|c|c|c|c|c|}
\hline $\mathrm{N}=167$ & \multicolumn{2}{|c|}{1} & \multicolumn{2}{|c|}{2} & \multicolumn{2}{|c|}{3} & \multicolumn{2}{|c|}{4} & \\
\hline Grupo & $\mathrm{F}$ & $\%$ & $\mathrm{~F}$ & $\%$ & $\mathrm{~F}$ & $\%$ & $\mathrm{~F}$ & $\%$ & Media & D.Típ \\
\hline A & 0 & 0 & 9 & 20.5 & 25 & 56.8 & 10 & 22.7 & 3.02 & 0.664 \\
\hline $\mathrm{B}$ & 1 & 1.9 & 8 & 15.4 & 31 & 59.6 & 12 & 23.1 & 3.04 & 0.685 \\
\hline C & 0 & 0 & 6 & 8.5 & 35 & 49.3 & 30 & 42.3 & 3.34 & 0.631 \\
\hline Total & 1 & 0.6 & 23 & 13.8 & 91 & 54.5 & 52 & 31.1 & 3.16 & 0.671 \\
\hline
\end{tabular}

En cuanto a la utilización de las TIC y la Web 2.0 en general, un 92.2\% afirmó que tras realizar el curso de guitarra abogaban por el uso estas herramientas y plata- 
formas como forma de potenciar el proceso de enseñanza-aprendizaje musical (Tabla 6). Los resultados por grupos presentaron valores similares.

Tabla 6. El proceso de enseñanza-aprendizaje musical potenciado por el uso de las TIC y la Web 2.0.

\begin{tabular}{|l|c|c|c|c|c|c|c|c|c|c|}
\hline $\mathrm{N}=167$ & \multicolumn{2}{|c|}{1} & \multicolumn{2}{|c|}{2} & \multicolumn{2}{|c|}{3} & \multicolumn{2}{|c|}{4} & & \\
\hline Grupo & $\mathrm{F}$ & $\%$ & $\mathrm{~F}$ & $\%$ & $\mathrm{~F}$ & $\%$ & $\mathrm{~F}$ & $\%$ & Media & D. Típ \\
\hline A & 1 & 2.3 & 5 & 11.4 & 27 & 61.4 & 11 & 25.0 & 3.09 & 0.676 \\
\hline B & 0 & 0 & 6 & 11.5 & 39 & 75.0 & 7 & 13.5 & 3.02 & 0.505 \\
\hline C & 0 & 0 & 1 & 1.4 & 34 & 47.9 & 36 & 50.7 & 3.49 & 0.531 \\
\hline Total & 1 & 0.6 & 12 & 7.2 & 100 & 59.9 & 54 & 32.3 & 3.24 & 0.603 \\
\hline 1- Muy en
\end{tabular}

1= Muy en desacuerdo; $2=$ En desacuerdo; $3=$ De acuerdo; $4=$ Totalmente de acuerdo

Con respecto al número y a la distribución de las sesiones de este curso, un 51.5\% consideró que tanto el número como el calendario de sesiones no permitía la asimilación de los contendidos propuestos (Tabla 7). Las diferencias intergrupales fueron evidentes, debido a que en los Grupos B y C el porcentaje de participantes que consideró inapropiado el número y distribución de sesiones (65.3\% y 67.6\%, respectivamente) era mucho menor que en el Grupo A (90.0\%).

Tabla 7. Número de sesiones presenciales y distribución temporal satisfactoria.

\begin{tabular}{|l|c|c|c|c|c|c|c|c|c|c|}
\hline $\mathrm{N}=167$ & \multicolumn{2}{|c|}{1} & \multicolumn{2}{|c|}{2} & \multicolumn{2}{|c|}{3} & \multicolumn{2}{|c|}{4} & \\
\hline Grupo & $\mathrm{F}$ & $\%$ & $\mathrm{~F}$ & $\%$ & $\mathrm{~F}$ & $\%$ & $\mathrm{~F}$ & $\%$ & Media & D.Típ \\
\hline A & 17 & 38.6 & 23 & 52.3 & 4 & 9.1 & 0 & 0 & 1.70 & 0.632 \\
\hline B & 6 & 11.5 & 28 & 53.8 & 17 & 32.7 & 1 & 1.9 & 2.25 & 0.682 \\
\hline C & 13 & 18.3 & 35 & 49.3 & 19 & 26.8 & 4 & 5.6 & 2.20 & 0.804 \\
\hline Total & 36 & 21.6 & 86 & 51.5 & 40 & 24.0 & 5 & 3.0 & 2.08 & 0.756 \\
\hline 1= Muy en desacuerdo; 2= En desacuerdo; 3= De acuerdo; 4= Totalmente de acuerdo &
\end{tabular}

En lo referente al componente innovador de la metodología utilizada en el curso, un $85.1 \%$ estuvo de acuerdo o totalmente de acuerdo en considerarla como innovadora (Tabla 8). Si bien todos los porcentajes grupales fueron altos, en el caso del Grupo C fue muy elevado, pues alcanzó el $98.6 \%$ de sus participantes.

Tabla 8. Metodología innovadora.

\begin{tabular}{|l|c|c|c|c|c|c|c|c|c|c|}
\hline $\mathrm{N}=167$ & \multicolumn{3}{|c|}{1} & \multicolumn{2}{|c|}{2} & \multicolumn{2}{|c|}{3} & \multicolumn{2}{|c|}{4} & \\
\hline Grupo & $\mathrm{F}$ & $\%$ & $\mathrm{~F}$ & $\%$ & $\mathrm{~F}$ & $\%$ & $\mathrm{~F}$ & $\%$ & Media & D.Típ \\
\hline $\mathrm{A}$ & 2 & 4.5 & 11 & 25.0 & 27 & 61.4 & 4 & 9.1 & 2.75 & 0.686 \\
\hline $\mathrm{B}$ & 2 & 3.8 & 9 & 17.3 & 34 & 65.4 & 7 & 13.5 & 2.88 & 0.676 \\
\hline $\mathrm{C}$ & 0 & 0 & 1 & 1.4 & 46 & 64.8 & 24 & 33.8 & 3.32 & 0.501 \\
\hline Total & 4 & 2.4 & 21 & 12.6 & 107 & 64.1 & 35 & 21.0 & 3.04 & 0.658 \\
\hline
\end{tabular}


En relación con las expectativas iniciales cumplidas por parte del alumnado, dos tercios de los participantes (67.1\%) sintieron cumplidas las expectativas que tenían antes de hacer este curso (Tabla 9). Los resultados por grupos constataron discrepancias considerables entre ellos. En el Grupo A, menos de la mitad del alumnado (43.2\%) estuvo de acuerdo o totalmente de acuerdo con haber cumplido las expectativas iniciales del curso, mientras que el Grupo C alcanzó el 78.9\% en esas categorías.

Tabla 9. Expectativas iniciales cumplidas.

\begin{tabular}{|l|c|c|c|c|c|c|c|c|c|c|}
\hline N=167 & \multicolumn{2}{|c|}{1} & \multicolumn{2}{|c|}{2} & \multicolumn{2}{|c|}{3} & \multicolumn{2}{|c|}{4} & & \\
\hline Grupo & F & $\%$ & F & $\%$ & F & $\%$ & F & $\%$ & Media & D.Típ \\
\hline A & 7 & 15.9 & 18 & 40.9 & 19 & 43.2 & 0 & 0 & 2.27 & 0.727 \\
\hline B & 1 & 1.9 & 14 & 26.9 & 33 & 63.5 & 4 & 7.7 & 2.77 & 0.614 \\
\hline C & 1 & 1.4 & 14 & 19.7 & 48 & 67.6 & 8 & 11.3 & 2.89 & 0.599 \\
\hline Total & 9 & 5.4 & 46 & 27.5 & 100 & 59.9 & 12 & 7.2 & 2.69 & 0.685 \\
\hline
\end{tabular}

1= Muy en desacuerdo; $2=$ En desacuerdo; $3=$ De acuerdo; $4=$ Totalmente de acuerdo

En cuanto a la relevancia que puede tener el aprendizaje de la guitarra en el futuro desarrollo profesional de los participantes, un $67.7 \%$ estuvo de acuerdo o totalmente de acuerdo con la proyección importante que tendría dicho aprendizaje en su ulterior labor docente en el aula de Educación Infantil (Tabla 10). Los valores obtenidos por el Grupo A fueron muy inferiores a los alcanzados por los otros dos grupos, debido a que menos de la mitad de su alumnado $(43,2 \%)$ consideró que el curso de iniciación a la guitarra tendría una importante proyección en su profesión, mientras que en los grupos $\mathrm{B}$ y $\mathrm{C}$ dicho porcentaje casi se duplicaba ( $73 \% \mathrm{y}$ $78.9 \%$, respectivamente).

Tabla 10. Proyección importante en el desarrollo futuro de la docencia en Educación Infantil.

\begin{tabular}{|l|c|c|c|c|c|c|c|c|c|c|}
\hline $\mathrm{N}=167$ & \multicolumn{2}{|c|}{1} & \multicolumn{2}{|c|}{2} & \multicolumn{2}{|c|}{3} & \multicolumn{2}{|c|}{4} & \\
\hline Grupo & $\mathrm{F}$ & $\%$ & $\mathrm{~F}$ & $\%$ & $\mathrm{~F}$ & $\%$ & $\mathrm{~F}$ & $\%$ & Media & D.Típ \\
\hline A & 2 & 4.5 & 23 & 52.3 & 19 & 43.2 & 0 & 0 & 2.39 & 0.579 \\
\hline $\mathrm{B}$ & 1 & 1.9 & 13 & 25.0 & 32 & 61.5 & 6 & 11.5 & 2.83 & 0.648 \\
\hline $\mathrm{C}$ & 0 & 0 & 15 & 21.1 & 49 & 69.0 & 7 & 9.9 & 2.89 & 0.549 \\
\hline Total & 3 & 1.8 & 51 & 30.5 & 100 & 59.9 & 13 & 7.8 & 2.74 & 0.623 \\
\hline 1= Muy en desacuerdo; 2= En desacuerdo; 3 De acuerdo; 4= Totalmente de acuerdo \\
\hline
\end{tabular}

Finalmente, es importante mencionar que más de dos tercios de los participantes $(71.8 \%)$ se consideraron capacitados para acompañar canciones infantiles con la guitarra (Tabla 11). Los resultados alcanzados por los grupos son muy diferentes, debido a que en los Grupos B y C más del $80 \%$ del alumnado se sentía competente para realizar acompañamientos sencillos, mientras que en el Grupo A dicho valor se reduce a la mitad (40.8\%). 
Tabla 11. Capacidad para acompañar canciones infantiles con la guitarra.

\begin{tabular}{|c|c|c|c|c|c|c|c|c|c|c|}
\hline $\mathrm{N}=167$ & \multicolumn{2}{|c|}{1} & \multicolumn{2}{|c|}{2} & \multicolumn{2}{|c|}{3} & \multicolumn{2}{|c|}{4} & \multirow[b]{2}{*}{ Media } & \multirow[b]{2}{*}{ D.Típ } \\
\hline Grupo & $\mathrm{F}$ & $\%$ & $\mathrm{~F}$ & $\%$ & $\mathrm{~F}$ & $\%$ & $\mathrm{~F}$ & $\%$ & & \\
\hline A & 8 & 18.2 & 18 & 40.9 & 14 & 31.8 & 4 & 9.1 & 2.32 & 0.883 \\
\hline B & 1 & 1.9 & 8 & 15.4 & 38 & 73.1 & 5 & 9.6 & 2.90 & 0.569 \\
\hline $\mathrm{C}$ & 1 & 1.4 & 11 & 15.5 & 48 & 67.6 & 11 & 15.5 & 2.97 & 0.609 \\
\hline Total & 10 & 6.0 & 37 & 22.2 & 100 & 59.8 & 20 & 12.0 & 2.78 & 0.731 \\
\hline
\end{tabular}

\subsection{Aspectos docentes}

Tras la puesta en práctica de este programa fundamentado en la utilización de videotutoriales, la figura del docente no fue considerada como prescindible para la adquisición de conocimientos por dos tercios del alumnado participante (67.7\%), si bien el tercio restante otorgaba al docente un rol secundario (Tabla 12). Los resultados por grupos no mostraron valores con diferencias importantes.

Tabla 12. Rol secundario del docente en el proceso de enseñanza-aprendizaje.

\begin{tabular}{|l|c|c|c|c|c|c|c|c|c|c|}
\hline $\mathrm{N}=167$ & \multicolumn{2}{|c|}{1} & \multicolumn{2}{|c|}{2} & \multicolumn{2}{|c|}{3} & \multicolumn{2}{|c|}{4} & \\
\hline Grupo & $\mathrm{F}$ & $\%$ & $\mathrm{~F}$ & $\%$ & $\mathrm{~F}$ & $\%$ & $\mathrm{~F}$ & $\%$ & Media & D.Típ \\
\hline A & 5 & 11.4 & 23 & 52.3 & 13 & 29.5 & 3 & 6.8 & 2.32 & 0.771 \\
\hline $\mathrm{B}$ & 6 & 11.5 & 27 & 51.9 & 18 & 34.6 & 1 & 1.9 & 2.27 & 0.689 \\
\hline $\mathrm{C}$ & 18 & 25.4 & 34 & 47.9 & 18 & 25.4 & 1 & 1.4 & 2.03 & 0.755 \\
\hline Total & 29 & 17.4 & 84 & 50.3 & 49 & 29.3 & 5 & 3.0 & 2.18 & 0.747 \\
\hline
\end{tabular}

En lo referente al nivel de exigencia ejercida por el docente, un 78.5\% consideró que el profesorado había sido exigente o muy exigente. No se apreciaron valores diferenciados por grupos (Tabla 13).

Tabla 13. La figura del docente me ha resultado: exigente-poco exigente.

\begin{tabular}{|l|c|c|c|c|c|c|c|c|c|c|}
\hline $\mathrm{N}=167$ & \multicolumn{2}{|c|}{1} & \multicolumn{2}{|c|}{2} & \multicolumn{2}{|c|}{3} & \multicolumn{2}{|c|}{4} & \\
\hline Grupo & $\mathrm{F}$ & $\%$ & $\mathrm{~F}$ & $\%$ & $\mathrm{~F}$ & $\%$ & $\mathrm{~F}$ & $\%$ & Media & D.Típ \\
\hline A & 0 & 0 & 4 & 9.1 & 27 & 61.4 & 13 & 29.5 & 3.20 & 0.594 \\
\hline $\mathrm{B}$ & 0 & 0 & 8 & 15.4 & 35 & 67.3 & 9 & 17.3 & 3.02 & 0.577 \\
\hline C & 3 & 4.2 & 21 & 29.6 & 40 & 56.3 & 7 & 9.9 & 2.72 & 0.701 \\
\hline Total & 29 & 17.4 & 102 & 61.1 & 33 & 19.8 & 3 & 1.8 & 2.06 & 0.665 \\
\hline
\end{tabular}

Por último, la capacidad motivadora del docente fue valorada por un $76 \%$ del alumnado como motivadora o muy motivadora (Tabla 14). Se apreciaron grandes 
diferencias entre el Grupo A (media 2.16) y los Grupos B y C (medias 3.21 y 3.48, respectivamente).

Tabla 14. La figura del docente me ha resultado: motivadora-desmotivadora.

\begin{tabular}{|l|c|c|c|c|c|c|c|c|c|c|}
\hline $\mathrm{N}=167$ & \multicolumn{2}{|c|}{1} & \multicolumn{2}{|c|}{2} & \multicolumn{2}{|c|}{3} & \multicolumn{2}{|c|}{4} & & \\
\hline Grupo & $\mathrm{F}$ & $\%$ & $\mathrm{~F}$ & $\%$ & $\mathrm{~F}$ & $\%$ & $\mathrm{~F}$ & $\%$ & Media & D. Típ \\
\hline A & 11 & 25.0 & 19 & 43.2 & 10 & 22.7 & 4 & 9.1 & 2.16 & 0.914 \\
\hline B & 0 & 0 & 6 & 11.5 & 29 & 55.8 & 17 & 32.7 & 3.21 & 0.637 \\
\hline C & 0 & 0 & 4 & 5.6 & 29 & 40.8 & 38 & 53.5 & 3.48 & 0.606 \\
\hline Total & 11 & 6.6 & 29 & 17.4 & 68 & 40.7 & 59 & 35.3 & 3.04 & 0.890 \\
\hline
\end{tabular}

1= Muy desmotivadora; 2 = Desmotivadora; $3=$ Motivadora; 4 Muy motivadora

\subsection{Análisis de los datos obtenidos en la entrevista}

Las categorías establecidas a partir de las unidades de significado planteadas por los entrevistados han sido: valoración de la experiencia como docentes basada en la planificación, diseño y utilización de unos materiales TIC propuestos por otro docente; planificación del programa de intervención; diseño y estructuración de los videotutoriales; y proceso de enseñanza-aprendizaje. A continuación se presenta la información obtenida agrupada en los mismos bloques que los resultados del cuestionario.

\section{Aspectos generales del programa}

En primer lugar y en relación con el número y distribución temporal de las sesiones planteadas en la planificación del programa de intervención, los profesores participantes valoraron la necesidad de no espaciar demasiado las sesiones: (P.1- Yo creo que una semana o 10 dias viene bien) (P2.- No ha beneficiado espaciar tanto las sesiones).

En relación con la carga de contenido proporcionada, las opiniones fueron contrarias entre los dos profesores: (P1.- Nueve acordes y tres rasgueos es una miseria, no creo que sea excesivo), (P2.- Para el que nace de cero, creo que es demasiado teniendo en cuenta las limitaciones que tenemos). En este sentido, los profesores señalaron la posibilidad de mantener los contenidos y aumentar a diez el número de sesiones, con una distribución de quince días a lo largo de todo el curso académico. En cuanto a la progresión de los contenidos, uno de los profesores resaltó: (P1.- La introducción y la recapitulación de los vídeos muy bien. Solo hubo en un momento la progresión de los contenidos en el que en una sesión en concreto no funcionaba y a la siguiente sí).

Con respecto al diseño de los videotutoriales, los participantes valoraron de forma igualitaria y positivamente la estructura de los videotutoriales (introducción, desarrollo y recapitulación). En relación con el discurso y la forma de comunicar las ideas, los profesores lo valoraron satisfactoriamente: (P1.- Muy bien, didáctica lenta y progresiva) (P2.- Bien lentitos). La calidad de imagen y de audio fue calificada de muy buena por parte de los dos profesores (P2.- La calidad de audio y vídeo están 
muy bien), al igual que la ajustada duración en cada uno de los videotutoriales (P1.La duración de los vídeos no era excesiva; P2.- No son ni cortos ni largos, muy bien).

\section{Aspectos docentes}

Con respecto a si el proceso de enseñanza-aprendizaje de la guitarra se había visto potenciado por el uso de estas TIC, se observó un pensamiento positivo generalizado por parte de los entrevistados: (P1.-Sin duda favorecen el aprendizaje), (P2.- La predisposición es mayor al aprendizaje de la guitarra con el uso de los videotutoriales. Tienen un profesor en casa).

En lo referente a la valoración de esta experiencia como docentes basada la planificación, diseño y utilización de unos materiales TIC propuestos por otro docente, las respuestas fueron positivas: (P1.- Uno tiene que hacerse poco a poco con la dinámica. Los materiales están muy bien organizados y los vídeos son muy buenos didácticamente), (P2.- Te pones unos zapatos que nos son los tuyos, pero es lo normal. El material es muy válido).

Finalmente, en cuanto a una posible elección de estos recursos multimedia audiovisuales para volver a impartir sus clases, la respuesta fue común entre los entrevistados: (P1.- Este recurso funciona y lo volvería a utilizar potenciando la metodología, [...] creo que ha faltado un pequeño plan de acción, una demanda de interactividad de actividades con los alumnos), (P.2- Sin duda esta metodología flipped classroom beneficia el proceso de enseñanza y la incorporaré en el futuro).

\section{Conclusiones}

Explorada la percepción del alumnado participante sobre el programa diseñado, se ha constatado una gran aceptación de la guitarra como instrumento para acompañar las canciones en el aula de infantil por parte de los participantes (80.9\%). Este hecho reafirma el planteamiento que consideraba la guitarra como un instrumento muy apropiado para el profesorado de Educación Infantil. Por otro lado, casi tres cuartas partes de la totalidad del alumnado constataron sentir cumplidas sus expectativas iniciales $(67.1 \%)$ y se mostraron favorables ante la proyección del aprendizaje adquirido en su futura labor docente $(67.7 \%)$. En consecuencia, un mayor número de participantes se sintieron capaces para acompañar canciones infantiles con la guita$\operatorname{rra}(71.8 \%)$.

En relación con el proceso de enseñanza-aprendizaje, prácticamente la totalidad del alumnado (95.8\%) consideró indispensables la utilización de los videotutoriales en el aprendizaje de la guitarra. Igualmente, nueve de cada diez participantes opinaron que los videotutoriales habían favorecido su formación musical e instrumental y abogaban por el uso de estas plataformas y herramientas para potenciar el proceso de enseñanza-aprendizaje musical. Por este motivo, podría considerarse que la inclusión de dichas herramientas en los planteamientos educativos musicales de docentes universitarios disminuye los efectos provocados por las limitaciones temporales y la falta de conocimientos previos mencionados anteriormente en este trabajo (Tejada, 2004; Romero y Vela, 2014; Palazón, 2015). Además, un propósito esencial en el 
diseño de este programa era favorecer que el alumnado estuviera motivado ante la ardua tarea de iniciarse en el dominio de un instrumento musical desconocido. En este sentido y coincidiendo con otros estudios (Navarro, Lavigne y Martínez, 2009; Palazón, 2013), los videotutoriales han ejercido un papel fundamental en la motivación de los participantes, quienes constataron la gran motivación generada por el uso de los videotutoriales (85.6\%). De igual forma, la figura del docente fue considerada motivadora por tres cuartas partes de la muestra, así como la metodología utilizada $(85.1 \%)$.

En cuanto al desarrollo del programa, la valoración fue bastante unánime al no estar de acuerdo con el número de sesiones presenciales, al igual que con su distribución a lo largo del curso. Esta aportación se corrobora con la opinión aportada por los docentes que pusieron en práctica el Curso de iniciación a la guitarra en Educación Infantil, quienes valoraron la necesidad de aumentar la frecuencia y el número de sesiones. En realidad era un resultado esperado si se tiene en cuenta la dificultad que presenta la iniciación a un instrumento como la guitarra en solo seis sesiones presenciales de clase. La valoración del programa ha servido para que el equipo docente de la asignatura haya optado por incrementar en un $50 \%$ el número de sesiones destinadas al aprendizaje de la guitarra.

Con respecto al rol del profesorado, en este estudio se pretendía que el profesorado adquiriera un segundo plano en el proceso de aprendizaje. Si bien un tercio de los participantes otorgó al docente un rol secundario, los dos tercios restantes consideraron indispensable su aportación en este proceso educativo. Estos resultados desmitifican la idea de que los recursos multimedia podrían sustituir la figura del profesor (Juvonen y Ruismäki, 2009; Giráldez, 2010) y ayuda a entender que la combinación de ambos elementos pueden ser la clave del éxito. Asimismo y al igual que en otros contextos educativos musicales, cuando se diseñó este programa se consideró que el profesorado debía promover en el alumnado un clima de esfuerzo y superación en la realización de sus propias producciones musicales. Un 78.5\% confirmó la exigencia ejercida por los docentes. Aunque este resultado ha sido considerado como un elemento positivo en el desarrollo del programa, los niveles de exigencia deben ser seleccionados diligentemente, pues un exceso de requerimientos podría provocar en los estudiantes la desmotivación y el abandono.

Por último, en algunos de los aspectos evaluados se ha observado una gran discrepancia de resultados entre los tres grupos de clase participantes, que en casos puntuales, como ha sido la valoración de la motivación aportada por el docente, puede oscilar entre muy motivadora (Grupo C) y desmotivadora (Grupo A). Dichas diferencias apuntan directamente a una variable presente en todos los programas de intervención educativa como es el profesorado. Nuevamente, los resultados reafirman la idea de que si bien la utilización de los materiales multimedia puede favorecer considerablemente el aprendizaje instrumental/musical del alumnado, la figura del profesor es crucial para que dichos recursos adquieran una importante dimensión educativa y sus potencialidades se transfieran efectivamente al discente (Area, 2007; Giráldez, 2012; Espigares et al., 2014). Por lo tanto, si realmente se quiere mejorar el proceso de enseñanza-aprendizaje musical, ambos recursos, humanos y materiales, deben ser sometidos a un profundo análisis y reflexión que aporte nuevas perspectivas y panorámicas sobre cómo se forma musicalmente a las futuras maestras y maestros de Educación Infantil. La introducción de la guitarra como instrumento acompañante solo es el inicio de una cadena de cambios que la formación 
del profesorado de infantil reclama desde hace años, que invita a desarrollar más investigaciones al respecto.

\section{Referencias bibliográficas}

Area, M. (2007). Algunos principios para el desarrollo de buenas prácticas pedagógicas con las TICs en el aula. Comunicación y pedagogía: Nuevas tecnologías y recursos didácticos, 222, 42-47

Bautista, F. (2000). La metodología audiovisual como alternativa a la enseñanza instrumental tradicional. Revista de la Lista Electrónica Europea de Música en la Educación, 5, 1-6. Recuperado de musica.rediris.es/leeme/revista/bautista00.pdf

Bengochea, L., Domínguez, M., y Díez, T. (2014). La percepción de los docentes del uso didáctico de los videotutoriales accesibles. En Actas del V Congreso Internacional sobre Calidad y Accesibilidad de la Formación Virtual (CAFVIR 2014) (pp. 27-34). Disponible en: https:// goo.gl/UO3Iko.

Bernal, J. (1996). Implicaciones de la música en el currículum de educación infantil y formación de educadores. Tesis doctoral. Universidad de Málaga, España.

Capriles, I. (2016). El "guitarró" en el aula de educación infantil y primaria: una herramienta para la inclusión a través de la música. Tesis doctoral. Universidad de las Islas Baleares, España.

Espigares, M., García, R., Tejada, J., y Rebollo, M. A. (2014). El discurso del profesorado de Educación Musical en la innovación educativa con TIC. Posicionamientos en la evaluación del software Tactus. Revista Electrónica Complutense de Investigación en Educación Musical, 11, 1-16. doi: 10.5209/rev_RECIEM.2014.v11.43054

Gifford, E. (1993). The musical training of primary teachers: Old problems, new insights and possible solutions. British Journal of Music Education, 10, 33-46. doi: 10.1017/ S0265051700001418

Giles, A., \& Frego, R. (2004). An inventory of musical activities used by elementary classroom teachers: An exploratory study. Update: Applications of Research in Music Education, 22, 13-22. doi: 10.1177/87551233040220020103

Giráldez, A. (2009). Reflexiones en torno al lugar de las artes en la Educación Infantil. CEE Participación Educativa, 12, 100-109.

Giráldez, A. (2010). Repensar la educación musical en un mundo digital. En A. Giráldez (Ed.), Música. Complementos de formación disciplinar (pp. 73 $\square 100$ ). Barcelona: Graó.

Giráldez, A. (2012). La enseñanza musical online tiene un futuro prometedor. Revista Red Educativa Musical. Recuperado de http://recursostic.educacion.es/artes/rem/web/index. php/es/dossier-educativo/item/405-entrevista-sergio-blardony

Jambrina, E. (2007) La flauta dulce en el área de Expresión artística de Educación Primaria. Comunidad Autónoma de Extremadura. Realidad, implicación y propuestas para el profesorado. Tesis doctoral. Universidad de Extremadura, España.

Juvonen, A., \& Ruismäki, H. (2009). The new Horizons for Music Technology in Music Education. The Changing Face of Music and Art Education, 1, 98-104.

Lockett, W. (2010). Student perceptions about the effectiveness and quality of on line musical instrument instruction. Tesis doctoral. Capella University, Estados Unidos.

López, I, y Lago, P. (2013). El aprendizaje online de un instrumento musical. Revista Didáctica, Innovación y Multimedia, 27, 1-15. Recuperado de http://dim.pangea.org/ revistaDIM27/revista27OCmusical.htm 
López de la Calle, M. (2009). La formación de los maestros de Educación Infantil para la comprensión de la música y su uso didáctico en Galicia. REIFOP, 12(1), 107-120.

Lorenzo, J. (1995). Perspectiva histórica de la formación de los maestros en España (13701990). Revista Complutense de Educación, 2(6), 203-229.

McMillan, J., y Schumacher, S. (2005). Investigación educativa. Madrid: Pearson Educación.

Miles, M.B., \& Huberman, A.M. (1994). Qualitative Data Analysis: An expanded sourcebook. Thounsand Oaks, CA: Sage Publications.

Mills, J. (2005). Music in the school. Oxford: Oxford University Press.

Navarro, J., Lavigne, G., y Martínez, G. (2009). Curso de guitarra clásica en línea: blogs para la enseñanza musical. Revista Electrónica de LEEME, 24, 23-48. Recuperado de http:// musica.rediris.es/leeme/revista/navarroetal09.pdf

Orden ECI/3854/2007, de 27 de diciembre, por la que se establecen los requisitos para la verificación de los títulos oficiales que habiliten para el ejercicio de la profesión de Maestro de Educación Infantil. Boletín Oficial del Estado, 312, de 29 de diciembre de 2007, pp. $53735-53738$.

Palazón, J. (2013). El podcasting: una tecnología web 2.0 para el apoyo y la mejora de la interpretación instrumental del alumnado de música en la Educación secundaria obligatoria. Tesis doctoral. Universidad de Murcia, España.

Palazón, J. (2014). De youtube al vodcasting: la web 2.0 de vídeo como propulsora de nuevos escenarios. En Actas II Congreso Virtual Internacional sobre Innovación Pedagógica y Praxis Educativa (pp. 40-49). Sevilla

Palazón, J. (2015). Internet como plataforma para la enseñanza y aprendizaje musical: audiovisuales online para la práctica instrumental. Conferencia presentada en el XXII Congreso Internacional sobre Educación y Aprendizaje, Madrid, España. Recuperado de: https://www.researchgate.net/publication/275646953_Internet_como_plataforma_para_la enseanza_y_aprendizaje_musical_audiovisuales_online_para_la_prctica_instrumental

Reeves, T. (2000). Enhancing the Worth of Instructional Technology Research through "Design Experiments" and Other Development Research Strategies. International Perspectives on Instructional Technology Research for the 21st Century Symposium. New Orleans, Estados Unidos.

Reeves, T. (2006). Design research from the technology perspective. En J. Van den Akker, K. Gravemeijer, S. McKenney, \& N. Nieveen (Eds.), Educational design research (pp. 86-109). London: Routledge. doi: 10.4324/9780203088364

Ródenas, M. (2012). La utilización de los videotutoriales en educación. Ventajas e inconvenientes. Sofware gratuito en el mercado. Revista Digital Sociedad de la Información 33, 1-9. Recuperado de http://www.sociedadelainformacion.com/33/videos. pdf

Romero, S., y Vela, M. (2014). Edublogs musicales en el tercer ciclo de educación primaria: perspectiva de alumnos y profesores. Revista Complutense de Educación, 25(1), 195 221. doi: 10.5209/rev_RCED.2014.v25.n1.41351

Ruiz, E., y Santamaría, R. (2013). La formación musical en el profesorado de Educación Infantil. Estudios sobre el Mensaje Periodístico, 19, 439448. doi: 10.5209/rev_ ESMP.2013.v19.42051

Serrano, F.J. (1999). Análisis de relatos. En J. Sáez, A. Escarbajal, A. García y M. Campillo (Coords.), Cuentos pedagógicos, relatos educativos (pp. 33-71). Murcia: Diego Marín.

Shoemaker, K., \& Van Stam, G. (2010). e-Piano, a Case of Music Education via internet in Rural Zambia. In G. Schreiber et.al. (Eds.), Proceedings of the WebSci10: Extending the Frontiers of Society On-Line, Raleigh, NC: USA. 
Tejada, J. (2004). Música y mediación de la tecnología en sus procesos de aprendizaje. Educación XX1,7, 15-26.

Upitis, R., Brook, J., \& Abrami, P. (2014). Enhancing music learning with digital tools: A case study of a student using iSCORE. Journal of Literature and Arts Studies, 4, 489-497.

Vannatta-Hall, J. (2010). Music education in early childhood teacher education: The impact of a music methods course on pre-service teachers perceived confidence and competence to teach music. Dissertation Doctor of Education in Music Education. University of Illinois at Urbana-Champaingn, Estados Unidos. Recuperado de: https://www.ideals. illinois.edu/bitstream/handle/2142/16854/1_VannattaHall_Jennifer.pdf?sequence $=4$ 


\section{ANEXO 1}

Ejemplo de planificación de una sesión del Curso de iniciación a la guitarra en Educación Infantil

\begin{tabular}{|c|c|}
\hline SESIÓN 1 & KEEP CALM AND PLAY THE GUITAR \\
\hline OBJETIVOS & CONTENIDOS \\
\hline $\begin{array}{l}\text { - Conocer las partes más representativas de la guitarra } \\
\text { - Adoptar correctamente la posición para tocar } \\
\text { - Afinar correctamente la guitarra con tunerr.com u } \\
\text { - Aprender la digitación de la mano izquierda y la for- } \\
\text { ma cóncava de la mano derecha } \\
\text { - Reconocer la simbología utilizada para los rasgueos } \\
\text { - Aprender el acorde C y (Am) opcional }\end{array}$ & $\begin{array}{l}\text { - Cifrado americano } \\
\text { - Cuerdas al aire de la guitarra para afinar correctamen- } \\
\text { te y para las notas de referencia } \\
\text { - Partes de la guitarra } \\
\text { - Posición de la guitarra } \\
\text { - Las manos en la guitarra } \\
\text { - Afinación de la guitarra } \\
\text { - Acordes: C y (Am) }\end{array}$ \\
\hline
\end{tabular}

- Visionado del videotutorial "Sesión 1" donde se tratan los contenidos anteriores (VT.1)

- Afinar la guitarra con el afinador online www.tunerr.com o aplicación móvil

- Leer y comprender el material teórico SESIÓN 1 (DT.1)

\section{DESARROLLO DE LA CLASE (60’)}

1. Preguntas iniciales

- ¿Habéis tenido algún problema para visionar los videotutoriales?

- ¿Habéis comprendido todos los contenidos de los vídeos?

- ¿Habéis tenido algún problema o duda con los contenidos?

- ¿Habéis podido afinar correctamente la guitarra con el afinador online o aplicación?

2. Verificación de la afinación de las guitarras

Dividir la clase en subgrupos y comprobar cuerda por cuerda si están bien afinadas.

3. Actividad de recordatorio

Repasar los contenidos de los vídeos relacionados con las partes de la guitarra, la posición para tocar el instrumento y la digitación de los dedos.

4. Acordes

Enseñar y practicar el acorde de $\mathrm{C}$ y el $1^{\text {er }}$ rasgueo binario. Explicar la nomenclatura de los acordes.

5. Interpretación de canciones

Canción: Vamos a palmear

- Interpretación de toda la canción a modo de ejemplo por parte del profesorado.

- Todo el grupo interpreta la canción muy lentamente con el acorde de C, marcando con el pulgar hacia abajo con el acorde correspondiente al principio de cada compás.

- Mostrar cómo obtener la nota de referencia para poder cantar la canción.

- Toda la clase entona y acompaña la canción muy lentamente.

6. Actividad de ampliación

Si el grupo realiza la canción anterior con facilidad, se explicará el acorde de

Am y se trabajará la canción ¿Dónde estás?

\begin{tabular}{|l|l|}
\hline \multicolumn{1}{|c|}{ DOCUMENTOS } & \multicolumn{1}{c|}{ VIDEOS } \\
\hline - Material Teórico Sesión 1 (DT.1) & $\bullet$ Video Tutorial Sesión 1 (VT.1) \\
\hline
\end{tabular}

